Pacific Journal of Mathematics

AN ANALOGUE OF THE WIENER-TAUBERIAN THEORE
FOR SPHERICAL TRANSFORMS ON SEMISIMPLE LIE 


\section{AN ANALOGUE OF THE WIENER-TAUBERIAN THEOREM FOR SPHERICAL TRANSFORMS ON SEMI-SIMPLE LIE GROUPS}

\section{Alladi Sitaram}

Let $G$ be a semi-simple connected noncompact Lie group with finite center and $K$ a fixed maximal compact subgroup of $G$. Fix a Haar measure $d x$ on $G$ and let $I_{1}(G)$ denote those functions in $L^{1}(G, d x)$ which are biinvariant under $K$. The purpose of this paper is to prove that if $f \in I_{1}(G)$ is such that its spherical Fourier transform (i.e., Gelfand transform) $\hat{f}$ is nowhere vanishing on the maximal ideal space of $I_{1}(G)$ and $\hat{f}$ "does not vanish too fast at $\infty$ ", then the ideal generated by $f$ is dense in $I_{1}(G)$. This generalizes earlier results of Ehrenpreis-Mautner for $G=\mathrm{SL}(2$, $R$ ) and R. Krier for $G$ of real rank one.

1. Introduction. Let $f$ be an $L^{1}$-function on $\boldsymbol{R}$ (or more generally on a locally compact abelian group). Then the celebrated Wiener-Tauberian theorem says that if the Fourier transform $\hat{f}$ is a nowhere vanishing function then the ideal generated by $f$ is dense in $L^{1}(\boldsymbol{R})$. In [1] Ehrenpreis and Mautner observe that the corresponding result is not true if one considers the commutative Banach algebra of $K$-biinvariant functions on noncompact semisimple Lie group $G$, where $K$ is a maximal compact subgroup of $G$. More precisely, let $G=\mathrm{SL}(2, \boldsymbol{R})$ i.e., the group of $2 \times 2$ real matrices of determinant 1 , and

$$
K=\mathrm{SO}(2)=\left\{\left(\begin{array}{rr}
\cos \theta & \sin \theta \\
-\sin \theta & \cos \theta
\end{array}\right) ; 0 \leqq \theta \leqq 2 \pi\right\} \text { and let }
$$

$I_{1}(G)$ denote the commutative Banach algebra of $K$-biinvariant $L^{1}$-functions on $G$. For $f \in I_{1}(G)$, let $\hat{f}$ denote its spherical Fourier transform (see §2). Then Ehrenpreis and Mautner observed that there exist functions $f \in I_{1}(G)$ such that $\hat{f}$ does not vanish anywhere on the maximal ideal space of $I_{1}(G)$ and yet the algebra generated by $f$ is not dense in $I_{1}(G)$. However they were able to show that if $\hat{f}$ is non vanishing and $\hat{f}$ 'does not go to zero too fast at $\infty$ ' then the ideal generated by $f$ is indeed dense in $I_{1}(G)$. (Theorems 6 and 7 of [1].) These results have been generalized by R. Krier [6] in his thesis when $G$ is a noncompact connected semi-simple Lie group of real rank 1 . (The author does not know whether Krier's results have been published.) The purpose of this note is to prove a theorem in the spirit of Theorem 7 of [1] without any restriction 
on the rank of $G$. While the basic technique we use is that of [1], we have to use the more recent results of Trombi-Varadarajan [7] and some observations of Gangolli-Warner [4] to prove our main theorem. Indeed in [3] Gangolli predicts that a theorem of the Trombi-Varadarajan type would yield a Tauberian type theorem.

2. Notation and preliminaries. (For any unexplained notation and terminology please see [5].) $G$ will denote a connected noncompact semi-simple lie group with finite center and $K$ a fixed maximal compact subgroup of $G$. Fix an Iwasawa decomposition $G=\mathrm{KAN}$ and let $\boldsymbol{a}$ be the Lie algebra of $A$. Let $\boldsymbol{a}^{*}$ be the real dual of $a$ and $a^{*}$ its complexification. Let $\rho$ be the half-sum of the positive roots for the adjoint action of $\boldsymbol{a}$ on $\boldsymbol{g}$ (where $\boldsymbol{g}$ is the Lie algebra of $G)$. The Killing form will induce a form $\langle\cdot, \cdot\rangle$ on $a^{*} \times a^{*}$. Then, as is well known, $\langle\cdot, \cdot\rangle$ is positive definite on $\boldsymbol{a}^{*} \times \boldsymbol{a}^{*}$. Extend the form $\langle\cdot, \cdot\rangle$ to a bilinear form on $\boldsymbol{a}_{c}^{*} \times \boldsymbol{a}_{c}^{*}$. This bilinear form also will be denoted by $\langle\cdot, \cdot\rangle$. Let $W$ be the Weyl group of the symmetric space $G / K$. Then there is a natural action of $W$ on $\boldsymbol{a}$, $\boldsymbol{a}^{*}$ and $\boldsymbol{a}_{\mathrm{c}}^{*}$ and $\langle\cdot, \cdot\rangle$ is invariant under the action of $W$.

For each $\lambda \in \boldsymbol{a}_{c}^{*}$ let $\phi_{\lambda}$ be the elementary spherical function associated with $\lambda$. (Recall that $\phi_{\lambda}$ is given by the formula, $\phi_{\lambda}(x)=$ $\int_{K} e^{(i \lambda-\rho)(I I(x k))} d k$ - see [5] for details.) Then it is known that $\phi_{\lambda}=\phi_{\lambda}^{\prime}$, iff $\exists s \in W$ with $s \lambda=\lambda^{\prime}$. Let $F=\left\{\lambda ; \phi_{\lambda}\right.$ is a bounded function on $\left.G\right\}$. Then it is known (a theorem of Helgason and Johnson) that:

$$
F=\boldsymbol{a}^{*}+i C_{\rho} \text { where } C_{\rho}=\text { convex hull of }\{s \rho: s \in W\} \text {. }
$$

Let $P\left(\boldsymbol{a}_{c}^{*}\right)$ be the symmetric algebra over $\boldsymbol{a}_{c}^{*}$. Then each $u \in P\left(\boldsymbol{a}_{c}^{*}\right)$ gives rise to a differential operatior $\partial(u)$ on $\boldsymbol{a}_{c}^{*}$.

Let $I(G)$ be the set of all complex valued spherical functions on $G$, i.e., $I(G)=\left\{f ; f\left(k_{1} x k_{2}\right)=f(x), k_{1}, k_{2} \in K, x \in G\right\}$. Fix a Haar measure $d x$ on $G$ and let $I_{1}(G)=I(G) \cap L^{1}(G)$. Then it is well known that $I_{1}(G)$ is a commutative Banach algebra under convolution (and that the maximal ideal space of $I_{1}(G)$ can be identified with $F / W$ ). We shall denote by $I^{\infty}(G)$ the space of $C^{\infty}$-spherical functions and by $I_{c}^{\infty}(G)$ the space of compactly supported functions in $I^{\infty}(G)$.

For $f \in I_{1}(G)$ define its spherical Fourier transform, $\hat{f}$ on $F$ by:

$$
\widehat{f}(\lambda)=\int_{G} f(x) \phi_{-\lambda}(x) d x, \quad \lambda \in F .
$$

Then it is known that $\hat{f}$ is a $W$-invariant bounded function on $F$, holomorphic in $F^{0}$ (=interior of $F$ ) and continuous on $F$. Also $(f * g)^{\wedge}=\hat{f} \cdot \hat{g}$ for $f, g \in I_{1}(G)$ where $f * g$ is the convolution of $f$ and $g$ and is given by 


$$
(f * g)(y)=\int_{G} f\left(y x^{-1}\right) g(x) d x, \quad y \in G .
$$

If $f \in I_{c}^{\infty}(G)$ then $\hat{f}$ is defined on all of $\boldsymbol{a}_{c}^{*}$ (and in fact will be an entire $W$-invariant function on $\boldsymbol{a}_{c}^{*}$ satisfying the Paley-Wiener growth condition-see [2]).

We shall now introduce a space of rapidly decreasing functions in $I^{\infty}(G)$ which we will denote by $S_{1}(G)$. (This is the so called $L^{1}$-Harish-Chandra-Schwartz space of spherical functions):

Let $x \in G$. Then $x=k \quad \exp X, k \in K, X \in \boldsymbol{p} \quad(\boldsymbol{g}=\boldsymbol{k}+\boldsymbol{p}$ is the Cartan decomposition of the Lie algebra $g$ of $G$ ). Put $\sigma(x)=\|X\|$, where $\|\cdot\|$ is the norm induced on $\boldsymbol{p}$ by the restriction of the Killing form. For any left invariant differential operator $D$ on $G$ and any integer $r \geqq 0$, we define for $f \in I^{\infty}(G)$

$$
p_{D, r}(f)=\sup _{x \in G}(1+\sigma(X))^{r}\left|\phi_{0}(x)\right|^{-2}|D f(x)|
$$

where $\phi_{0}$ is the elementary spherical function corresponding to $\lambda=0$. Define $S_{1}(G)=\left\{f ; f \in I^{\infty}(G)\right.$ and $\left.p_{D, r}(f)<\infty \forall r, D\right\} . \quad S_{1}(G)$ becomes a Frechet-space when equipped with topology induced by the family of semi norms $p_{D, r}$. It is known that $S_{1}(G) \hookrightarrow I_{1}(G)$ and $I_{c}^{\infty}(G) \hookrightarrow S_{1}(G)$ are both dense inclusions.

Now let $Z(F)$ be the space of functions $f$ on $F$ satisfying the following conditions: (i) $f$ is holomorphic in $F^{0}$ and continuous on $F$, (ii) If $u \in P\left(\boldsymbol{a}_{c}^{*}\right)$ and $l \geqq 0$ is any integer, then $q_{u, l}(f)=$ $\sup _{\lambda \in F^{0}}\left(1+\|\lambda\|^{2}\right)^{l}|(\partial(u) f)(\lambda)|<\infty$, (where $\|\lambda\|^{2}=\left\|\lambda_{1}\right\|^{2}+\left\|\lambda_{2}\right\|^{2}, \lambda=$ $\lambda_{1}+i \lambda_{2}, \lambda_{1}, \lambda_{2} \in a^{*}$ and $\left.\left\|\lambda_{i}\right\|^{2}=\left\langle\lambda_{i}, \lambda_{i}\right\rangle\right)$. Let $\bar{Z}(F)$ denote the subspace of $Z(F)$ consisting of $W$-invariant functions. $Z(F), \bar{Z}(F)$ are algebras under pointwise multiplication and we topologize them by the family of semi norms $q_{u l}$. In this topology $Z(F), \bar{Z}(F)$ are Frechet spaces. If $a \in \bar{Z}(F)$ define the 'wave packet' $\psi_{a}$ on $G$ by:

$$
\psi_{a}(x)=\frac{1}{|W|} \int_{a^{*}} a(\lambda) \dot{\phi}_{\lambda}(x) c(\lambda)^{-1} c(-\lambda)^{-1} d \lambda,
$$

( $|W|$ is the order of the Weyl group).

$(c(\lambda)$ is the well known $c$-function of Harish-Chandra and one knows that $c(\lambda)^{-1} c(-\lambda)^{-1}$ is a continuous function on $a^{*}$ of at most polynomial growth. Further if $d \mu$ is the measure on $a^{*}$ defined by $d \mu=|W|^{-1} c(\lambda)^{-1} c(-\lambda)^{-1} d \lambda$, then one knows that the map $f \rightarrow \hat{f}$ is an isometry of $I(G) \cap L^{2}(G)$ onto $L^{2}\left(a^{*}, d \mu\right)^{W}$ where the superscript $W$ indicates Weyl-group invariants in $L^{2}\left(a^{*}, d \mu\right)$ ). We are now finally in a position to state the theorem of Trombi-Varadarajan [7]:

THEOREM 2.1. (i ) If $f \in S_{1}(G)$, then $\hat{f} \in \bar{Z}(F)$. 
(ii) If $a \in \bar{Z}(F)$ then the integral defining the wave packet $\psi_{a}$ converges absolutely and in fact $\psi_{a} \in S_{1}(G)$ and $\hat{\psi}_{a}=a$.

(iii) The map $f \rightarrow \hat{f}$ is a topological linear isomorphism of $S_{1}(G)$ onto $\bar{Z}(F)$.

Before closing this section we introduce some more function spaces and state a proposition due to Gangolli-Warner [4]. (As the authors point out in [4] this proposition can be obtained by a careful examination of the proof of Theorem 2.1 of Trombi-Varadarajan.)

Let $m, l$ be nonnegative integers and let us put $\bar{Z}_{m, l}(F)$ for the space of functions $f$ on $F$ such that (i) $f$ is holomorphic in $F^{0}$, continuous on $F$, and invariant under the action of $W$ (ii) If $u \in P\left(\boldsymbol{a}_{c}^{*}\right)$ and degree $u \leqq m$, then

$$
q_{u, l}(f)=\sup _{\lambda \in F^{0}}\left(1+\|\lambda\|^{2}\right)^{l}|(\partial(u) f)(\lambda)|<\infty .
$$

Put $\bar{Z}_{m}(F)=\bigcap_{l \geq 0} \bar{Z}_{m, l}(F)$. Then tne following proposition is contained in Proposition 3.3 and Corollary 3.4 of Gangolli-Warner [4].

Proposition 2.2. Let $G$ be a noncompact connected semi-simple Lie group with finite center. Then $\exists$ an integer $m_{G}$ (depending only on the group $G)$ such that if $a \in \bar{Z}_{m_{G}}(F)$, then:

(i) The integral defining the wave packet $\psi_{a}$ converges absolutely.

(ii) $\psi_{a} \in I_{1}(G)$.

3. An analogue of the Wiener-Tauberian theorem. Before we state and prove the main theorem we will first prove a couple of preliminary lemmas which will be used in the proof of the main theorem. The first lemma is a very mild strengthening of Proposition 2.2 and the second lemma is a slight generalization of Lemma 5.2 for the case of $G=\operatorname{SL}(2, R)$ in [1].

LEMMA 3.1. There exists an integer $m_{G}$ (depending only on the group $G)$ such that if $a \in \bar{Z}_{m_{G}}(F)$ then all the following conditions are satisfied

(i) The integral defining $\psi_{a}$ (the wave packet) converges absolutely.

(ii) $\psi_{a} \in I_{1}(G)$.

(iii) $\hat{\psi}_{a}=a$.

Proof. From Proposition 2.2 it follows that we can find an integer $m_{G}$ such that if $a \in \bar{Z}_{m_{G}}(F)$ then (i) and (ii) are satisfied. We will show that (iii) is also satisfied. Observe first that if $a \in \bar{Z}_{m_{G}}(F)$, 
then since $(\forall l)$ it decays faster than $1 /\left(1+\|\lambda\|^{2}\right)^{l}$ on $a^{*}$ and since $c(\lambda)^{-1} c(-\lambda)^{-1}$ has at most polynomial growth, $a$ is integrable with respect to the measure $c(\lambda)^{-1} c(-\lambda)^{-1} d \lambda$ on $a^{*}$. To prove that $\hat{\psi}_{a}=a$, we first show that

$$
\begin{aligned}
\forall b \in \bar{Z}(F), \int_{a^{*}} a(\lambda) b(\lambda) c(\lambda)^{-1} c(-\lambda)^{-1} d \lambda \\
=\int_{a^{*}} \hat{\psi}_{a}(\lambda) b(\lambda) c(\lambda)^{-1} c(-\lambda)^{-1} d \lambda .
\end{aligned}
$$

The integral on the left hand side exists since both $a, b$ decay faster than $1 /\left(1+\|\lambda\|^{2}\right)^{l}$ on $a^{*}$ and $c(\lambda)^{-1} c(-\lambda)^{-1}$ has at most polynomial growth. The integral on the right hand side exists because $\hat{\psi}_{a}$ is a bounded function (being the spherical Fourier transform of an integrable function) and $b$ is a rapidly decreasing function. The proof of $\left(^{*}\right)$ is a straightforward application of Fubini's theorem keeping in mind the following facts (i) Since $b \in \bar{Z}(F), \dot{\psi}_{b} \in S_{1}(G)$ and is hence integrable and further $\hat{\psi}_{b}=b$ (ii) $\psi_{a}$ is an integrable function on $G$ and $a(\lambda)$ is integrable with respect to $c(\lambda)^{-1} c(-\lambda)^{-1} d \lambda$. Since $\left(^{*}\right)$ is true $\forall b \in \bar{Z}(F)$ and since $\bar{Z}(F)$ contains 'enough' functions it follows easily that

$$
a(\lambda) c(\lambda)^{-1} c(-\lambda)^{-1}=\hat{\psi}_{a}(\lambda) c(\lambda)^{-1} c(-\lambda)^{-1} \text { a.e. on } a^{*}
$$

with respect to Lebesgue measure. But the zeros of $c(\lambda)^{-1} c(-\lambda)^{-1}$ must have zero Lebesgue measure in $a^{*}$ and hence it follows that $a=\hat{\psi}_{a}$.

LEMMA 3.2. Let $k$ be a fixed nonnegative integer and let $\phi(z)=e^{\langle z, z\rangle}, z \in F$. Define $X$ by $X=\{h ; h \in \bar{Z}(F)$ and $h \phi \in \bar{Z}(F)\}$. Then $X$ is a dense linear subspace of $\bar{Z}(F)$.

Proof. Let $\psi_{n}(z)=e^{-\langle z, z\rangle^{k+1 / n}}$. Then since $\langle\cdot, \cdot\rangle$ is $W$-invariant, $\psi_{n}, \phi$ are $W$-invariant. It is easy to see that $\psi_{n}, \phi \psi_{n} \in \bar{Z}(F)$. (To see this observe that $F=a^{*}+i C_{\rho}$. Clearly $\psi_{n}, \phi \psi_{n}$ are rapidly decreasing on $\boldsymbol{a}^{*}$, but if $z \in F$ the 'imaginary' part of $z$ varies only over a compact set.) Hence if $f \in \bar{Z}(F), f \phi \psi_{n} \in \bar{Z}(F)$. Now it is easy to see that as $n \rightarrow \infty, f \psi_{n} \rightarrow f$ in the topology of $\bar{Z}(F)$. But since $f \psi_{n} \phi \in \bar{Z}(F), f \psi_{n} \in X$ and the lemma is proved.

We are now in a position to state and prove our main theorem.

THEOREM 3.3. Let $f \in I_{1}(G)$ and suppose

(i) $\hat{f}$ is nowhere vanishing on $F$.

(ii) $\exists$ a positive integer $k$ such that for every $u \in P\left(\boldsymbol{a}_{c}^{*}\right)$ with degree $u \leqq m_{G}$ (where $m_{G}$ is as in Lemma 3.1) we have 


$$
\sup _{z \in F^{0}}\left|\partial(u)\left[(\hat{f}(z))^{-1} e^{-\langle z, z\rangle^{k}}\right]\right|<\infty .
$$

Then the ideal generated by $f$ is dense in $I_{1}(G)$.

Proof. (Note: condition (ii) says that ' $\hat{f}$ does not vanish too fast at $\infty^{\prime}$.) Let $X$ be as in Lemma 3.2. Let $Y=\left\{\psi_{a} ; a \in X\right\}$. Since by Lemma $3.2 X$ is dense in $\bar{Z}(F)$, by Theorem 2.1, $Y$ is dense in $S_{1}(G)$. Hence since $S_{1}(G) \hookrightarrow I_{1}(G)$ is a dense inclusion, $Y$ is a dense subspace of $I_{1}(G)$. We will show that every $h \in Y$ can be written as $h=f * g$, with $g \in I_{1}(G)$ and this will prove the theorem. Now if $h \in Y, \hat{h} \in X$ and $\hat{h}=\hat{f} \cdot \hat{f}^{-1} \hat{h}$.

(Note that since $\hat{f}$ does not vanish on $F, \hat{f}^{-1}$ is well defined on $F$.)

Now we claim $\hat{f}^{-1} \hat{h}$ is in $\bar{Z}_{m_{G}}(F)$. This follows from the definition of $X$ and condition (ii) of Theorem 3.3 (since $\hat{f}(z)^{-1} h(z)=\hat{f}(z)^{-1}$ $\left.e^{-\langle z, z\rangle^{k}} e^{\langle z, z\rangle^{k}} \hat{h}(z)\right)$. Hence by Lemma $3.1 \psi \hat{\psi} \hat{f}-1 \hat{h} \in I_{1}(G)$ and $\hat{\psi} \hat{f}-1 \hat{k}=$ $\hat{f}^{-1} \hat{h}$.

Claim: $h=f * \psi_{\hat{f}-1 \hat{h}}$. This is because

$$
(f * \psi \hat{f}-1 \hat{h})^{\wedge}=\hat{f} \hat{\psi} \hat{f}-1 \hat{h}=\hat{f} \hat{f}^{-1} \hat{h}=\hat{h} .
$$

Hence (by the semi simplicity of $\left.I_{1}(G)\right) f * \psi_{\hat{f}-1 \hat{h}}=h$. Thus we have shown that every function $h$ in a dense subspace $Y$ of $I_{1}(G)$ can be writted as $h=f * g$ and this concludes the proof of our theorem.

(Note: For $G=\mathrm{SL}(2, \boldsymbol{R})$ or more generally for $G$ a real rank one group $m_{G}=2$ (see [1], [6]).)

4. The case of $L^{p}$ for $1 \leqq p \leqq 2$. For $\varepsilon \geqq 0$, let $F^{\varepsilon}=a^{*}+i \varepsilon C_{\rho}$. Then one can introduce the spaces $Z\left(F^{\varepsilon}\right), \bar{Z}\left(F^{\varepsilon}\right)$ just as in $\S 2$. Let $I_{p}(G)=I(G) \cap L^{p}(G)$. Then one can define the so called $L^{p}$-Harish Chandra-Schwartz subspace of $K$-biinvariant functions i.e., $S_{p}(G) \subseteq$ $I_{p}(G)$ (see [7] for details). Actually the theorem of Trombi-Varadarajan is more general than stated in $\S 2$. In fact they show that under the map $f \rightarrow \hat{f}$ the spaces $S_{p}(G)$ and $\bar{Z}\left(F^{\varepsilon}\right)$ where $\varepsilon=2 / p-1$ are topologically isomorphic $(p \leqq 2)$. Also one knows that if $p \geqq 1$ then $S_{1}(G) \hookrightarrow S_{p}(G)$ is a dense inclusion. Using this one can modify the arguments in the last section to obtain the following theorem.

THEOREM 4.1. Let $1 \leqq p<2$ and $f \in I_{p}(G) \cap I_{1}(G)$, such that:

(i) $\hat{f}$ is nowhere vanishing on $F$.

(ii) $\exists$ a positive integer $k$ such that for every $u \in P\left(a_{c}^{*}\right)$ with degree $u \leqq m_{G}\left(m_{G}\right.$ as in Lemma 3.1), we have

$$
\sup _{z \in F^{0}}\left|\partial(u)\left[(\hat{f}(z))^{-1} e^{-\langle z, z\rangle^{k}}\right]\right|<\infty \text {. }
$$


Then the set of functions of the form $g * f, g \in I_{c}^{\infty}(G)$, is dense in $I_{p}(G)$.

Finally we observe that the Plancharel theorem for $I_{2}(G)$ (i.e., the spherical Fourier transform is an isometric isomorphism of $I_{2}(G)$ onto $L^{2}\left(a^{*}, \mu\right)^{W}$, where the superscript indicates Weyl-group invariance and $\mu$ is the measure on $a^{*}$ defined by $\left.d \mu=|W|^{-1} c(\lambda)^{-1} c(-\lambda)^{-1} d \lambda\right)$ gives us the following fact: Let $f \in I_{2}(G)$ such that $\hat{f}$ is nonvanishing on $a^{*}$ except possibly on a set of $\mu$-measure zero. Then the set of functions of the form $g * f, g \in I_{c}^{\infty}(G)$ is dense in $I_{2}(G)$. (The proof of this fact is exactly as in the case of abelian groups).

ACKNOWLEDGMENT. The author would like to express his thanks to Professor V.S. Varadarajan for his interest in this work. Some suggestions of Professor M.S. Raghunathan were very helpful for some of the proofs. The author would also like to thank Dr. M.K.V. Murthy, Dr. S. C. Bagchi, Mr. S. Kumaresan and Mr. Adi Murthy for many stimulating conversations.

\section{REFERENCES}

1. L. Ehrenpreis and F. I. Mautner, Some properties of the Fourier transform on semi-simple Lie groups, I, Ann. of Math., 61 (1955), 406-439.

2. R. Gangolli, On the Plancharel formula and the Paley-Wiener theorem for spherical functions on semi-simple Lie groups, Ann. of Math., 93 (1971), 150-165.

3. - Spherical functions on semi-simple Lie groups, in-Symmetric Spaces, Editors-W. M. Boothby and G. L. Weiss, Marcel and Dekker, New York, 1972.

4. R. Gangolli and G. Warner, On Selberg's trace formula, J. Mathematical Soc. of Japan, 27 (1975), 328-344.

5. S. Helgason, Differential Geometry and Symmetric Spaces, Academic Press, New York, 1962.

6. R. Krier, Ph. D. thesis, University of Nancy, 1973.

7. P. C. Trombi and V. S. Varadarajan, Spherical transforms on semi-simple Lie groups, Ann. of Math., 94 (1971), 246-303.

Received January 9, 1979.

INDIAN STATISTICAL INSTITUTE 203, BarRackPoRe TRUNK ROAD CALCUTta 700 035, India 



\section{PACIFIC JOURNAL OF MATHEMATICS}

\section{EDITORS}

DONALD BABBITT (Managing Editor)

University of California

Los Angeles, CA 90024

HUGo RossI

University of Utah

Salt Lake City, UT 84112

C. C. MOORE and ANDREW OGG

University of California

Berkeley, CA 94720
J. DugundJI

Department of Mathematics

University of Southern California

Los Angeles, CA 90007

R. FinN and J. Milgram

Stanford University

Stanford, CA 94305

ASSOCIATE EDITORS
E. F. BECKENBACH
B. H. NeUmanN
F. WOLF
K. YoSHIDA

\section{SUPPORTING INSTITUTIONS}

UNIVERSITY OF BRITISH COLUMBIA

CALIFORNIA INSTITUTE OF TECHNOLOGY

UNIVERSITY OF CALIFORNIA

MONTANA STATE UNIVERSITY

UNIVERSITY OF NEVADA, RENO

NEW MEXICO STATE UNIVERSITY

OREGON STATE UNIVERSITY

UNIVERSITY OF OREGON
UNIVERSITY OF SOUTHERN CALIFORNIA

STANFORD UNIVERSITY

UNIVERSITY OF HAWAII

UNIVERSITY OF TOKYO

UNIVERSITY OF UTAH

WASHINGTON STATE UNIVERSITY

UNIVERSITY OF WASHINGTON

The Supporting Institutions listed above contribute to the cost of publication of this Journal, but they are not owners or publishers and have no responsibility for its content or policies.

Mathematical papers intended for publication in the Pacific Journal of Mathematics should be in typed form or offset-reproduced, (not dittoed), double spaced with large margins. Please do not use built up fractions in the text of the manuscript. However, you may use them in the displayed equations. Underline Greek letters in red, German in green, and script in blue. The first paragraph or two must be capable of being used separately as a synopsis of the entire paper. Please propose a heading for the odd numbered pages of less than 35 characters. Manuscripts, in triplicate, may be sent to any one of the editors. Please classify according to the scheme of Math. Reviews, Index to Vol. 39. Supply name and address of author to whom proofs should be sent. All other communications should be addressed to the managing editor, or Elaine Barth, University of California, Los Angeles, California, 90024.

50 reprints to each author are provided free for each article, only if page charges have been substantially paid. Additional copies may be obtained at cost in multiples of 50 .

The Pacific Journal of Mathematics is issued monthly as of January 1966. Regular subscription rate: $\$ 84.00$ a year (6 Vols., 12 issues). Special rato: $\$ 42.00$ a year to individual members of supporting institutions.

Subscriptions, orders for numbers issued in the last three calendar years, and changes of address shoud be sent to Pacific Journal of Mathematics, P.O. Box 969, Carmel Valley, CA 93924, U.S.A Old back numbers obtainable from Kraus Periodicals Co., Route 100, Millwood, NY 10546.

PUBLISHED BY PACIFIC JOURNAL OF MATHEMATICS, A NON-PROFIT CORPORATION

Printed at Kokusai Bunken Insatsusha (International Academic Printing Co., Ltd.). 8-8, 3-chome, Takadanobaba, Shinjuku-ku, Tokyo 160, Japan.

Copyright (C) 1980 by Pacific Jounal of Mathematics Manufactured and first issued in Japan 


\section{Pacific Journal of Mathematics \\ Vol. 89, No. $2 \quad$ June, 1980}

Frank Hayne Beatrous, Jr. and R. Michael Range, On holomorphic

approximation in weakly pseudoconvex domains................. 249

Lawrence Victor Berman, Quadratic forms and power series fields ...... 257

John Bligh Conway and Wacław Szymański, Singly generated antisymmetric operator algebras ....................... 269

Patrick C. Endicott and J. Wolfgang Smith, A homology spectral sequence for submersions . . . .................................

Sushil Jajodia, Homotopy classification of lens spaces for one-relator groups with torsion ................................ 301

Herbert Meyer Kamowitz, Compact endomorphisms of Banach

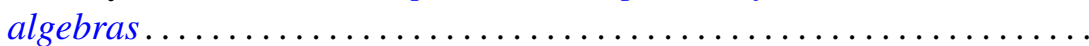

Keith Milo Kendig, Moiré phenomena in algebraic geometry: polynomial

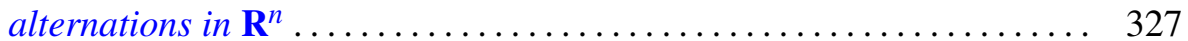

Cecelia Laurie, Invariant subspace lattices and compact operators....... 351

Ronald Leslie Lipsman, Restrictions of principal series to a real form . . . . . 367

Douglas C. McMahon and Louis Jack Nachman, An intrinsic

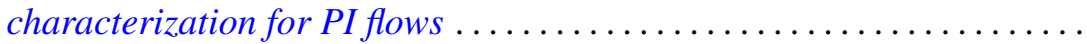

Norman R. Reilly, Modular sublattices of the lattice of varieties of inverse semigroups .................................... 405

Jeffrey Arthur Rosoff, Effective divisor classes and blowings-up of $\mathbf{P}^{2}$ 419

Zalman Rubinstein, Solution of the middle coefficient problem for certain

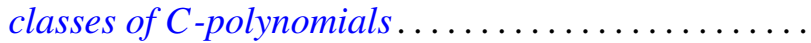

Alladi Sitaram, An analogue of the Wiener-Tauberian theorem for spherical transforms on semisimple Lie groups ................

Hal Leslie Smith, A note on disconjugacy for second order systems ...

J. Wolfgang Smith, Fiber homology and orientability of maps ...

Audrey Anne Terras, Integral formulas and integral tests for series of positive matrices. 